\title{
Assessment and Composition of Fish in the River District Maro Merauke
}

\author{
Edy H.P Melmambessy ${ }^{1}$, Irianis L. Latuperissa ${ }^{1}$, Marius Agustinus Welliken ${ }^{1}$, Nova Suryawati Monika ${ }^{1}$, Rosa \\ Delima Pangaribuan ${ }^{1}$.
}

${ }^{1}$ Departement Management of Aquatic Resource, Agriculture of Faculty, Musamus University

\begin{abstract}
Maro River is one of the major rivers in Merauke Regency that has a width of about 43-900 m and length of $300 \mathrm{~km}$. The purpose of this study is to find out the type and composition of fish caught using gill net in Maro River, Merauke Regency. This study was conducted in Maro River, Merauke Regency from May to June 2014. It was conducted using survey method. The data analysis included: species composition, relative abundance, availability frequency, diversity index and dominance index. The results of showed 1,084 fish in 17 species with the following composition; 490 of Kurtus gulliveri species (37,01\%), 212 of Eleutheronema tetradactylum species (20,33\%), 170 of Polydactylus plebeius species (16,30\%), 75 of crassilabris Cinetodus species (7,19\%), 64 of Cinetodus froggatti species (6,14\%), 37 of Argyrosomus amoyensis species (3,55\%), 20 of Mugil cephalus species (1,92\%), 15 of Megalops cyprinoides species (1,53\% ), 15 of Thryssa scratchleyi species (1,4\%), 12 of Lebtobrama sp species (1,15\%), 9 of Lates calcarifer species (0,86\%), 7 of Marilyna meraukensis species (0,67\%), 7 of Scomberomorus commerson species (0,67\% ), 5 of Nibea squamosal species (0,48\%), 4 of Rhinoptera javanica species $(0,38 \%), 3$ of Harpadon microchir species $(0,29 \%)$ and 1 of Datnioides campbelli species $(0,10 \%)$. Based on the availability frequency, there are 37 species with a percentage of $100 \%, 37$ species with $67 \%$ and 7 species with $33 \%$. The diversity of fish has a moderate value of $1<\mathrm{H}^{\prime}<3$. The obtained dominance index value is close to 0 , then it is stated that there is no species found to be dominant.
\end{abstract}

Keywords: River; Species Composition; Abundance; Availability; Diversity and Dominance.

\section{INTRODUCTION}

Merauke Regency has a brackish water resources potential of $22.204 \mathrm{Kg}$ [1]. The data concerning the number of potential local consumption of fish/Kg by type per District in Merauke Regency showed a total of 4.094.426 Kg [11]. The abundant natural resources are strongly supported by many rivers that flows into the Arafura sea, such as, Digul river, Bian river, Maro river and others. [2]

Maro River is one of the great rivers in Merauke that has a width of about 43-900 m and a length of 300 $\mathrm{km}$. This river has a historical value for Merauke, because the name Merauke derives from the name of this River. It can be interpreted that the characteristics of the area around the river is highly fertile, as it has great mangrove forests, and has mud substrate, so that the water turbidity is fairly high and has a relatively high water acidity as it is a meeting point between the freshwater and seawater along the river that becomes a unique habitat for fish and other living organisms contained in this river [3]. Based on the research conducted by [4]. there are many species of fish that exist in this Maro river, among others, eeltail catfish (Neosilurus $s p$ ), fly river gizzard shad (Nematalosa flyensis), oxeye herring (Megalops cyprinoids), nursery fish (Kurtus gulliveri), Belanak (Mugil sp) and fly river thryssa (Thryssa rastrosa).

$13 \%$ of the people in Gudang Arang, Maro Village, are fisherman who actively fishing. Fishing in the Maro River, especially for the Gudang Arang area, in the upstream, is an activity that has been done by people, especially for those living along the river [5]. Nevertheless, there is no vailable data in Merauke Regency about the composition of fish species caught, especially for Maro River in Gudang Arang. Therefore, it is necessary to conduct a study on the types and composition of fish to maintain and pay attention to its sustainability in nature and to be the basis of information about the types and composition of fish and sustainable management of fish resources.

Knowing the right to find out the types and composition of fish caught using gill net in Maro River in Gudang Arang, Maro Village, Merauke Regency.

\section{RESEARCH METHOD}

\subsection{Location and Time of Research}

The research is conducted from May to July 2014 at the Maro River in Gudang Arang, Maro Village, Merauke Regency.

\footnotetext{
Corresponding author: melmambessy@unmus.ac.id
} 


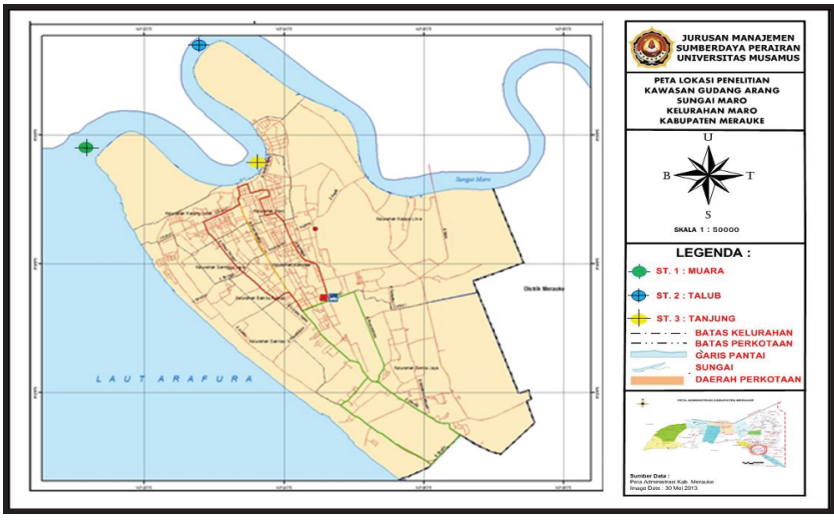

Fig 1. Map of Research Location (Maro River in Gudang Arang, Maro Village, Merauke Regency)

\subsection{Tools and Materials}

The tools used in this study were gill net (with a mesh size of 1 and 2 inch), boats, cool box, thermometer, $\mathrm{pH}$ meter, salinometer, analytical scales, hanging scales, plastic jars, plastic tray, tweezers, tissue, bucket , camera, stopwatch, fit bottle, raffia string, GPS, calculator, ruler, stationery, notebook and identification book. The materials used were ice cubes and types of caught fish.

\subsection{Research Method}

The method used in this research was survey method. Soehartono (1995) [6] suggests that the survey method is a method to obtain the existing data at the time of research conducted. This survey method can be a descriptive survey which aims to collect information a number of samples of people, object and others [7]. Such descriptive research uses survey method, while the data collection techniques used were as follows.

\subsubsection{Observation}

Sampling was conducted every Monday, Wednesday and Friday with 3 repetitions. The sampling technique used was purposive random sampling [8].

\subsubsection{Interview}

Interview is a data collection technique in survey methods that uses verbal questions to research subjects, which is conducted if the researcher needs communication to the respondents [6].

\subsection{Research Procedures}

\subsubsection{Measurement of physical factors, water chemistry}

Measuring the water temperature, flow velocity, salinity and water $\mathrm{pH}$.

\subsubsection{Collection of specimens or samples}

Sampling was done every Monday, Wednesday and Friday with 3 repetitions. The sample of fish was taken at low tide at three locations or stations, namely station 1 located in Estuary which is $\pm 5 \mathrm{~km}$ away before the meeting point with location II in Floodgate which is $\pm 5 \mathrm{~km}$ away after the meeting point with location III which is on the Cape. Fish sampling at each location was done at three points, i.e., the right, middle, and left bank of the river. In the right and left bank, fish sampling was conducted by using the gill net with a mesh size of 1 inch and in the middle bank by using the gill net with a mesh size of 2 inches.

The samples were collected in mesh pockets at each of the three stations: station I (Estuary), station II (Floodgate) and station III (Cape).

\subsubsection{Identification of Fish}

The identification was conducted by using manual identification (Allen, 1989, and Allen 1991) $[9,10]$ to determine the name of the collected species.

\subsection{Data Analysis}

The data analysis used descriptively is to process data obtained in field and compare with literature study. [16]

\subsubsection{Type Composition}

The type composition was obtained from the data of the size and number of nekton species obtained from each location with a predetermined station [12].

\subsubsection{Relative Abundance}

The calculation of relative abundance of each species was conducted by calculating the percentage of the number. The equation used was [13]

$$
\mathrm{Kr}=\frac{n i}{N} \times 100 \%
$$

Information :

$\mathrm{Kr}=$ relative abundance

$\mathrm{ni}=\mathrm{i}$-th individual species

$\mathrm{N}=$ total number of individuals of all species

\subsubsection{Availability Frequency}

Availability Frequency was used for showing the spread of local distribution of certain species. This is evident from the frequency (\%) of fish caught by using the following equation [14]

$$
F i=\frac{\boldsymbol{t i}}{\mathbf{T}} \times 100 \%
$$

\section{Information :}

$\mathrm{Fi}=$ availability frequency of $\mathrm{i}$-th caught species $(\%)$

$\mathrm{ti}=$ the number of stations in which the $\mathrm{i}$-th species is caught $\mathrm{T}=$ the number of all stations

\subsubsection{Diversity Index}

In order to determine the diversity of fish, it used the Shannon-Wiener index [15]:

$\mathrm{H}^{\prime}--\sum_{\mathrm{i}=1}^{n}\left(\frac{\mathbf{n i}}{\mathrm{N}}\right) \ln \left(\frac{n \mathrm{i}}{\mathrm{N}}\right)$

Information:

H' = Sahnnon -Wiener Diversity Index

$\mathrm{ni}=\mathrm{i}$-th individual species 
$\mathrm{N}=$ total number of individuals of all species

\subsubsection{Determination of Criteria:}

The ranges of diversity index value, according to Kreb (1989) are as follows: (1). H' $<1$ : low diversity, narrow spread of individual species and low community stability. (2). $1<\mathrm{H}^{\prime}<3$ : moderate diversity, moderate spread of individual species and moderate community stability. (3) $\mathrm{H}^{\prime}>3$ : high diversity, high spread of individual species and high community stability.

\subsubsection{Dominance Index}

In order to determine whether the fish are available, the Simpson's dominance index was used, as follow (Odum, 1996):

$$
\mathbf{C}=\sum\left(\frac{n i}{N}\right)^{2}
$$

Information:

C = Simpson's Diversity Index

$\mathrm{ni}=\mathrm{i}$-th individual species

$\mathrm{N}=$ total number of individuals of all species

Legendre, (1983) states the value of dominance index ranges from $0-1$; index 1 indicates very high dominance by one species (there is only one type in one station). While index 0 indicates that there is no dominance among the species found.

\section{RESULT AND DISCUSSION}

\subsection{Types of Fish Caught}

Table 1. Number of Species Caught per Station in The Research Location in Maro River, Gudang Arang, Maro Village, Merauke Regency

\begin{tabular}{|c|c|c|c|}
\hline \multirow[b]{2}{*}{ No } & \multicolumn{3}{|c|}{ Number of Species Caught in The Research Location } \\
\hline & $\begin{array}{l}\text { Station I } \\
\text { (Estuary) }\end{array}$ & $\begin{array}{c}\text { Station II } \\
\text { (Floodgate) }\end{array}$ & $\begin{array}{c}\text { Station III } \\
\text { (Cape) }\end{array}$ \\
\hline 1 & $\begin{array}{l}\text { Cinetodus } \\
\text { froggatti }\end{array}$ & $\begin{array}{l}\text { Cinetodus } \\
\text { froggatti }\end{array}$ & $\begin{array}{l}\text { Cinetodus } \\
\text { froggatti }\end{array}$ \\
\hline 2 & $\begin{array}{l}\text { Cinetodus } \\
\text { crassilabris }\end{array}$ & $\begin{array}{l}\text { Cinetodus } \\
\text { crassilabris }\end{array}$ & $\begin{array}{l}\text { Cinetodus } \\
\text { crassilabris }\end{array}$ \\
\hline 3 & Kurtus gulliveri & Kurtus gulliveri & $\begin{array}{l}\text { Kurtus } \\
\text { gulliveri }\end{array}$ \\
\hline 4 & $\begin{array}{l}\text { Argyrosomus } \\
\text { amoyensis }\end{array}$ & $\begin{array}{l}\text { Argyrosomus } \\
\text { amoyensis }\end{array}$ & $\begin{array}{l}\text { Argyrosomus } \\
\text { amoyensis }\end{array}$ \\
\hline 5 & $\begin{array}{l}\text { Polydactylus } \\
\text { plebeius }\end{array}$ & $\begin{array}{l}\text { Polydactylus } \\
\text { plebeius }\end{array}$ & $\begin{array}{l}\text { Polydactylus } \\
\text { plebeius }\end{array}$ \\
\hline 6 & $\begin{array}{l}\text { Eleutheronema } \\
\text { tetradactylum }\end{array}$ & $\begin{array}{l}\text { Eleutheronema } \\
\text { tetradactylum }\end{array}$ & $\begin{array}{l}\text { Eleutheronema } \\
\text { tetradactylum }\end{array}$ \\
\hline 7 & $\begin{array}{l}\text { Marilyna } \\
\text { meraukensis }\end{array}$ & $\begin{array}{l}\text { Marilyna } \\
\text { meraukensis }\end{array}$ & $\begin{array}{l}\text { Marilyna } \\
\text { meraukensis }\end{array}$ \\
\hline 8 & Mugil cephalus & & $\begin{array}{l}\text { Mugil } \\
\text { cephalus }\end{array}$ \\
\hline 9 & $\begin{array}{l}\text { Megalops } \\
\text { cyprinoides }\end{array}$ & & $\begin{array}{l}\text { Megalops } \\
\text { cyprinoides }\end{array}$ \\
\hline 10 & $\begin{array}{l}\text { Thryssa } \\
\text { scratchleyi }\end{array}$ & & $\begin{array}{l}\text { Thryssa } \\
\text { scratchleyi }\end{array}$ \\
\hline 11 & $\begin{array}{l}\text { Harpadon } \\
\text { microchir }\end{array}$ & & \\
\hline
\end{tabular}

\begin{tabular}{|c|c|c|c|}
\hline \multirow[b]{2}{*}{ No } & \multicolumn{3}{|c|}{ Number of Species Caught in The Research Location } \\
\hline & $\begin{array}{c}\text { Station I } \\
\text { (Estuary) }\end{array}$ & $\begin{array}{c}\text { Station II } \\
\text { (Floodgate) }\end{array}$ & $\begin{array}{c}\text { Station III } \\
\text { (Cape) }\end{array}$ \\
\hline 12 & $\begin{array}{l}\text { Datnioides } \\
\text { campbelli }\end{array}$ & & \\
\hline 13 & Nibea squamosal & & \\
\hline 14 & Lates calcarifer & & \\
\hline 15 & Lebtobrama sp & & \\
\hline 16 & $\begin{array}{l}\text { Rhinoptera } \\
\text { javanica }\end{array}$ & & \\
\hline 17 & $\begin{array}{l}\text { Scomberomorus } \\
\text { commerson }\end{array}$ & & \\
\hline $\begin{array}{c}\text { Total } \\
\text { Species }\end{array}$ & 17 & 7 & 10 \\
\hline
\end{tabular}

Based on Table 1, there were 17 fish species caught in Maro River in Gudang Arang. Based on Table 1, there were 17 fish species found in station I, 7 species found in station II and 10 species found in station III.

\subsection{Composition of Species}

Based on the results of research conducted in Maro River, Gudang Arang, Maro Village, Merauke Regency, there were 17 species with total composition of fish caught can be seen in Table 2 .

Table 2. Total fish caught in Maro River, Gudang Arang, Maro Village, Merauke Regency

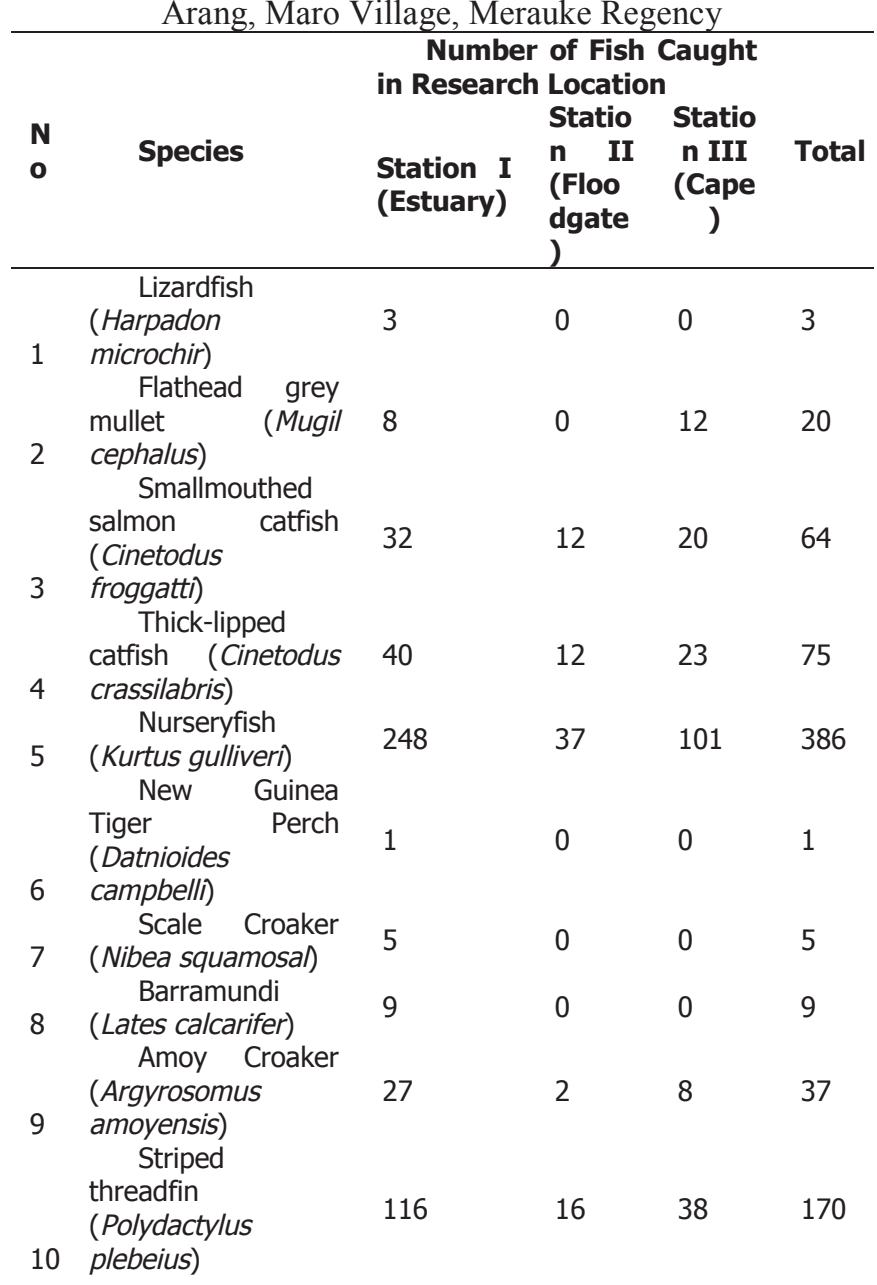




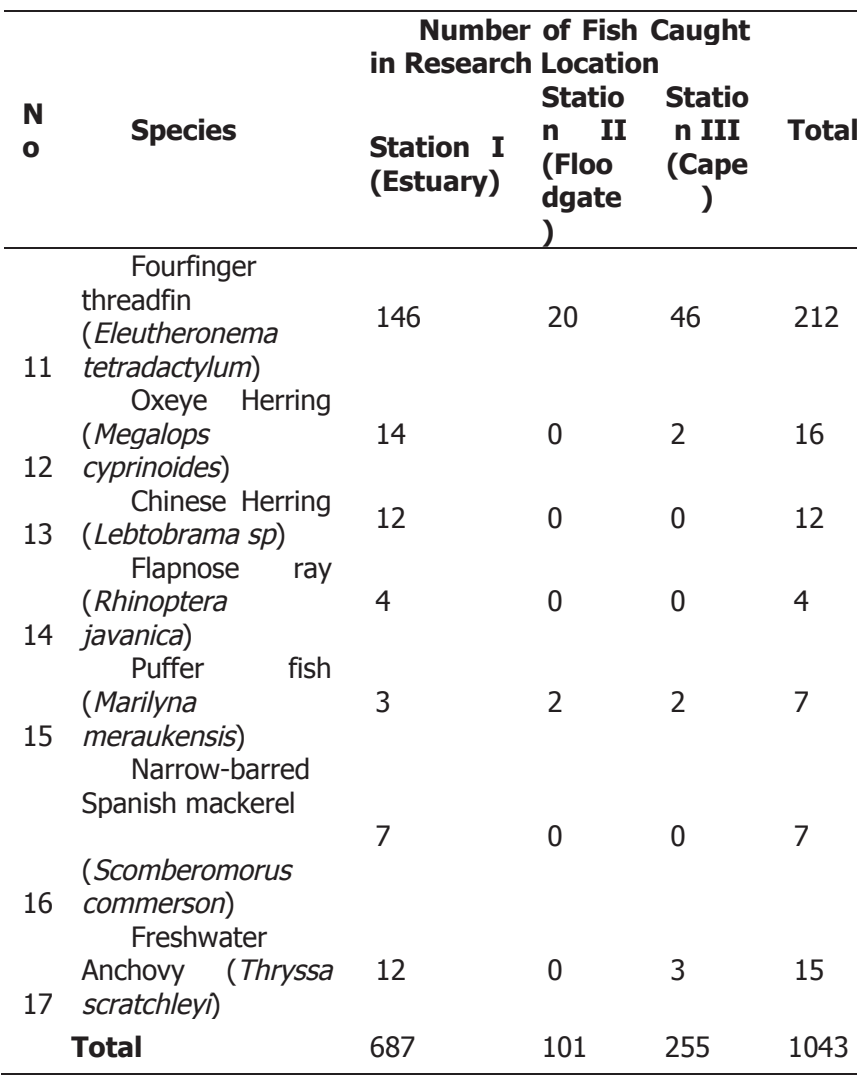

Based on the results (Table 2), the number of fish caught in 3 months (May to July 2014) in the Maro River, Gudang Arang, Maro Village, Merauke Regency, there were 1043 fish from 17 species spread over three research locations or stations. Where Station I (Estuary) is the location with the most fish caught of 687 and Unit III (Cape) is the location with the 2nd most fish caught of 101 and Unit II (Floodgate) is the location with the third most fish caught of 225 . Moreover, Kurtus gulliveri is the most type of fish caught in three stations with a total of 386.

\subsection{Relative Abundance}

Table 3. Percentage of Relative Abundance

\begin{tabular}{|c|c|c|c|c|c|}
\hline \multirow[b]{2}{*}{$\begin{array}{l}\mathbf{N} \\
\mathbf{0}\end{array}$} & \multirow[b]{2}{*}{ Species } & \multicolumn{3}{|c|}{$\begin{array}{l}\text { Percentage }(\mathbf{K r}) \text { of } \\
\text { Fish Caught in the } \\
\text { Research Location }(\%)\end{array}$} & \multirow[b]{2}{*}{$\begin{array}{l}\text { Total } \\
(\%)\end{array}$} \\
\hline & & $\begin{array}{l}\text { Station } \\
\text { I } \\
\text { (Estuar } \\
\text { y) }\end{array}$ & $\begin{array}{c}\text { Station } \\
\text { II } \\
\text { (Floodg } \\
\text { ate) }\end{array}$ & $\begin{array}{l}\text { itation } \\
\text { III } \\
\text { 'Cape) }\end{array}$ & \\
\hline 1 & $\begin{array}{l}\text { Lizardfish } \\
\text { (Harpadon } \\
\text { microchir) }\end{array}$ & 0,44 & 0 & 0 & 0,29 \\
\hline 2 & $\begin{array}{lr}\text { Flathead } & \text { grey } \\
\text { mullet } & \text { (Mugil } \\
\text { cephalus) }\end{array}$ & 1,16 & 0 & 4,71 & 1,92 \\
\hline 3 & $\begin{array}{l}\text { Smallmouthed } \\
\text { salmon catfish } \\
\text { (Cinetodus } \\
\text { froggatti) }\end{array}$ & 4,66 & 11,88 & 7,84 & 6,14 \\
\hline 4 & $\begin{array}{l}\text { Thick-lipped } \\
\text { catfish } \\
\text { (Cinetodus } \\
\text { crassilabris) }\end{array}$ & 5,82 & 11,88 & 9,02 & 7,19 \\
\hline 5 & $\begin{array}{l}\text { Nurseryfish } \\
\text { (Kurtus gulliveri) }\end{array}$ & 36,10 & 36,63 & 39,61 & 37,01 \\
\hline 6 & $\begin{array}{lr}\text { New } & \text { Guinea } \\
\text { Tiger } & \text { Perch }\end{array}$ & 0,15 & 0 & 0 & 0,10 \\
\hline
\end{tabular}

\begin{tabular}{|c|c|c|c|c|c|}
\hline \multirow[b]{2}{*}{$\begin{array}{l}\mathbf{N} \\
\mathbf{0}\end{array}$} & \multirow[b]{2}{*}{ Species } & \multicolumn{3}{|c|}{$\begin{array}{l}\text { Percentage }(\mathrm{Kr}) \text { of } \\
\text { Fish Caught in the } \\
\text { Research Location }(\%)\end{array}$} & \multirow[b]{2}{*}{$\begin{array}{l}\text { Total } \\
(\%)\end{array}$} \\
\hline & & $\begin{array}{l}\text { Station } \\
\text { I } \\
\text { (Estuar } \\
\text { y) }\end{array}$ & $\begin{array}{l}\text { Station } \\
\text { II } \\
\text { (Floodg } \\
\text { ate) }\end{array}$ & $\begin{array}{l}\text { Station } \\
\text { III } \\
\text { Cape) }\end{array}$ & \\
\hline & $\begin{array}{l}\text { (Datnioides } \\
\text { campbelli) }\end{array}$ & & & & \\
\hline 7 & $\begin{array}{l}\text { Scale Croaker } \\
\text { (Nibea } \\
\text { squamosal) }\end{array}$ & 0,73 & 0 & 0 & 0,48 \\
\hline 8 & $\begin{array}{l}\text { Barramundi } \\
\text { (Lates calcarifer) }\end{array}$ & 1,31 & 0 & 0 & 0,86 \\
\hline 9 & $\begin{array}{l}\text { Amoy Croaker } \\
\text { (Argyrosomus } \\
\text { amoyensis) }\end{array}$ & 3,93 & 1,98 & 3,14 & 3,55 \\
\hline 10 & $\begin{array}{l}\text { Striped threadfin } \\
\text { (Polydactylus } \\
\text { plebeius) }\end{array}$ & 16,89 & 15,84 & 14,90 & 16,30 \\
\hline 11 & $\begin{array}{l}\text { Fourfinger } \\
\text { threadfin } \\
\text { (Eleutheronema } \\
\text { tetradactylum) }\end{array}$ & 21,25 & 19,80 & 18,04 & 20,33 \\
\hline 12 & $\begin{array}{l}\text { Oxeye Herring } \\
\text { (Megalops } \\
\text { cyprinoides) }\end{array}$ & 2,04 & 0 & 0,78 & 1,53 \\
\hline 13 & $\begin{array}{l}\text { Chinese Herring } \\
\text { (Lebtobrama } s p \text { ) }\end{array}$ & 1,75 & 0 & 0 & 1,15 \\
\hline 14 & $\begin{array}{l}\text { Flapnose ray } \\
\text { (Rhinoptera } \\
\text { javanica) }\end{array}$ & 0,58 & 0 & 0 & 0,38 \\
\hline 15 & $\begin{array}{l}\text { Puffer fish } \\
\text { (Marilyna } \\
\text { meraukensis) }\end{array}$ & 0,44 & 1,98 & 0,78 & 0,67 \\
\hline 16 & $\begin{array}{l}\text { Narrow-barred } \\
\text { Spanish } \\
\text { mackerel }\end{array}$ & 1,02 & 0 & 0 & 0,67 \\
\hline & $\begin{array}{l}\text { (Scomberomorus } \\
\text { commerson) } \\
\text { Freshwater }\end{array}$ & & & & \\
\hline 17 & $\begin{array}{l}\text { Anchovy } \\
\text { (Thryssa } \\
\text { scratchleyi) }\end{array}$ & 1,75 & 0 & 1,18 & 1,44 \\
\hline & Total (\%) & 100 & 100 & 100 & 100 \\
\hline & Total (fish) & 687 & 101 & 255 & 1043 \\
\hline
\end{tabular}

Based on the results in Table 3 , the highest relative abundance is found in Nurseryfish (Kurtus gulliverI) in Station III with a percentage of $39,61 \%$. From the data in Table 3, the highest number of fish species caught is in station I with a total of 687 fish and the lowest is in station II with a total of 101 fish.

\subsection{Availability Frequency}

The Availability Frequency based on location of observation can be seen in Table 4.

Table 4. Percentage of Availability Frequency

\begin{tabular}{|c|c|c|c|c|c|}
\hline \multirow[b]{2}{*}{$\begin{array}{l}\mathbf{N} \\
\mathbf{0}\end{array}$} & \multirow[b]{2}{*}{ Species } & \multicolumn{2}{|c|}{$\begin{array}{l}\text { Availability } \\
\text { Frequency }\end{array}$} & \multirow[b]{2}{*}{$\begin{array}{c}\text { Statio } \\
\text { n III } \\
\text { (Cape } \\
\text { ) }\end{array}$} & \multirow[b]{2}{*}{$\begin{array}{l}\mathbf{F i} \\
(\%)\end{array}$} \\
\hline & & $\begin{array}{c}\text { Statio } \\
\text { n I } \\
\text { (Estu } \\
\text { ary) }\end{array}$ & $\begin{array}{c}\text { Station } \\
\text { II } \\
\text { (Floodg } \\
\text { ate) }\end{array}$ & & \\
\hline 1 & $\begin{array}{l}\text { Lizardfish } \\
\text { (Harpadon } \\
\text { microchir) }\end{array}$ & $\sqrt{ }$ & - & - & 33 \\
\hline 2 & Flathead & $\sqrt{ }$ & - & $\sqrt{ }$ & 67 \\
\hline
\end{tabular}




\begin{tabular}{|c|c|c|c|c|c|}
\hline \multirow[b]{2}{*}{$\begin{array}{l}\mathbf{N} \\
\mathbf{0}\end{array}$} & \multirow[b]{2}{*}{ Species } & \multicolumn{2}{|c|}{$\begin{array}{l}\text { Availability } \\
\text { Frequency }\end{array}$} & \multirow[b]{2}{*}{$\begin{array}{c}\text { Statio } \\
\text { n III } \\
\text { (Cape } \\
\text { ) }\end{array}$} & \multirow[b]{2}{*}{$\begin{array}{l}\mathbf{F i} \\
(\%)\end{array}$} \\
\hline & & $\begin{array}{l}\text { Statio } \\
\text { n I } \\
\text { (Estu } \\
\text { ary) }\end{array}$ & $\begin{array}{l}\text { Station } \\
\text { II } \\
\text { (Floodg } \\
\text { ate) }\end{array}$ & & \\
\hline & $\begin{array}{l}\text { mullet } \\
\text { cephalus) }\end{array}$ & & & & \\
\hline 3 & $\begin{array}{l}\text { Smallmouthed } \\
\text { salmon catfish } \\
\text { (Cinetodus } \\
\text { froggatti) }\end{array}$ & $\sqrt{ }$ & $\sqrt{ }$ & $\sqrt{ }$ & 100 \\
\hline 4 & $\begin{array}{l}\text { Thick-lipped } \\
\text { catfish (Cinetodus } \\
\text { crassilabris) }\end{array}$ & $\sqrt{ }$ & $\sqrt{ }$ & $\sqrt{ }$ & 100 \\
\hline 5 & $\begin{array}{l}\text { Nurseryfish } \\
\text { (Kurtus gulliveri) } \\
\text { New Guinea Tiger }\end{array}$ & $\sqrt{ }$ & $\sqrt{ }$ & $\sqrt{ }$ & 100 \\
\hline 6 & $\begin{array}{l}\text { Perch (Datnioides } \\
\text { campbelli) }\end{array}$ & $\sqrt{ }$ & - & - & 33 \\
\hline 7 & $\begin{array}{l}\text { Scale Croaker } \\
\text { (Nibea squamosal) }\end{array}$ & $\sqrt{ }$ & - & - & 33 \\
\hline 8 & $\begin{array}{l}\text { Barramundi (Lates } \\
\text { calcarifer) }\end{array}$ & $\sqrt{ }$ & - & - & 33 \\
\hline 9 & $\begin{array}{l}\text { Amoy Croaker } \\
\text { (Argyrosomus } \\
\text { amoyensis) }\end{array}$ & $\sqrt{ }$ & $\sqrt{ }$ & $\sqrt{ }$ & 100 \\
\hline 10 & $\begin{array}{l}\text { Striped threadfin } \\
\text { (Polydactylus } \\
\text { plebeius) }\end{array}$ & $\sqrt{ }$ & $\sqrt{ }$ & $\sqrt{ }$ & 100 \\
\hline 11 & $\begin{array}{l}\text { Fourfinger } \\
\text { threadfin } \\
\text { (Eleutheronema } \\
\text { tetradactylum) }\end{array}$ & $\sqrt{ }$ & $\sqrt{ }$ & $\sqrt{ }$ & 100 \\
\hline 12 & $\begin{array}{l}\text { Oxeye Herring } \\
\text { (Megalops } \\
\text { cyprinoides) }\end{array}$ & $\sqrt{ }$ & - & $\sqrt{ }$ & 67 \\
\hline 13 & $\begin{array}{l}\text { Chinese Herring } \\
\text { (Lebtobrama } s p \text { ) }\end{array}$ & $\sqrt{ }$ & - & - & 33 \\
\hline 14 & $\begin{array}{l}\text { Flapnose ray } \\
\text { (Rhinoptera } \\
\text { javanica) }\end{array}$ & $\sqrt{ }$ & - & - & 33 \\
\hline 15 & $\begin{array}{l}\text { Puffer } \quad \text { fish } \\
\text { (Marilyna } \\
\text { meraukensis) }\end{array}$ & $\sqrt{ }$ & $\sqrt{ }$ & $\sqrt{ }$ & 100 \\
\hline 16 & $\begin{array}{l}\text { Narrow-barred } \\
\text { Spanish mackerel } \\
\text { (Scomberomorus } \\
\text { commerson) } \\
\text { Freshwater }\end{array}$ & $\sqrt{ }$ & - & - & 33 \\
\hline 17 & $\begin{array}{l}\text { Anchovy (Thryssa } \\
\text { scratchleyi) }\end{array}$ & $\sqrt{ }$ & - & $\sqrt{ }$ & 67 \\
\hline
\end{tabular}

The results of Table 4 show the value of the availability frequency of the fish species in each station. The availability frequency value is related to the area of distribution. The greater the availability frequency value, the greater the area of distribution. The availability frequency value of fish species shows that there are 7 species with the percentage of $100 \%$ availability, means that the species have widespread distribution. Meanwhile, there are 3 species with a percentage of $67 \%$ availability, indicating moderate distribution and 7 species with a percentage of 33\% availability, indicating the low distribution.

\subsection{Diversity}

The diversity based on the observation location can be seen in Table 5 below.
Table 5. Diversity Index

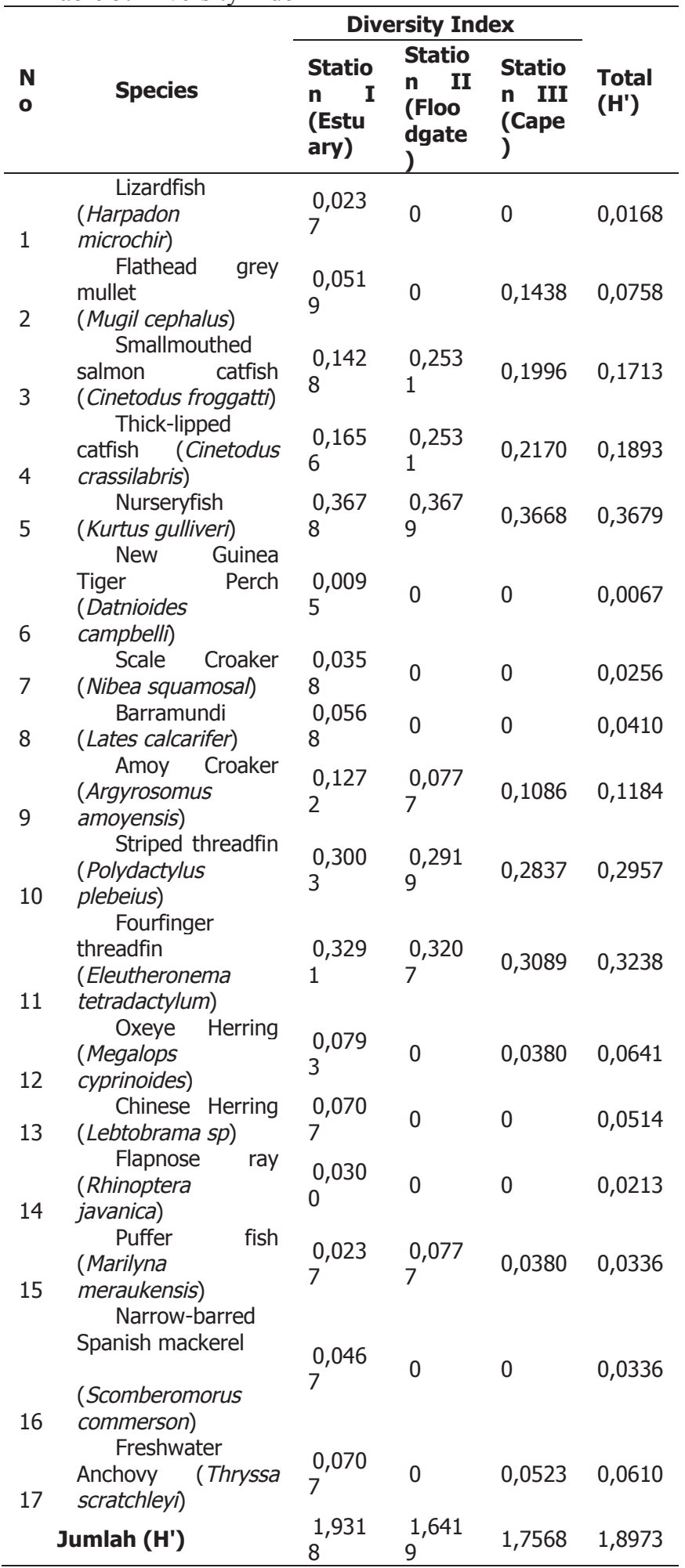

The diversity index value (Table 5) of species of fish caught for three months in Maro River, Gudang Arang, is 1,8973. This result indicates moderate diversity, moderate distribution of individual number of each species, and moderate community stability.

\subsection{Dominance}

The dominance index based on the observation location can be seen in Table 6 below

Table 6. Dominance Index 


\begin{tabular}{|c|c|c|c|c|c|}
\hline \multirow[b]{2}{*}{ No } & \multirow[b]{2}{*}{ Species } & \multicolumn{3}{|c|}{ Dominance Index } & \multirow[b]{2}{*}{$\begin{array}{l}\text { Total } \\
\text { (C) }\end{array}$} \\
\hline & & $\begin{array}{l}\text { Station I } \\
\text { (Estuary) }\end{array}$ & $\begin{array}{l}\text { Station II } \\
\text { (Floodgate) }\end{array}$ & $\begin{array}{l}\text { Station } \\
\text { III } \\
\text { (Cape) }\end{array}$ & \\
\hline 1 & $\begin{array}{l}\text { Lizardfish } \\
\text { microchir) }\end{array}$ & 0,00001936 & 0 & 0 & 0,00000841 \\
\hline 2 & $\begin{array}{l}\text { Flathead grey mullet } \\
\text { (Mugil cephalus) }\end{array}$ & 0,00013456 & 0 & $1^{0,0022184}$ & 0,00036864 \\
\hline 3 & $\begin{array}{l}\text { Smallmouthed salmon } \\
\text { catfish } \quad \text { (Cinetodus } \\
\text { froggatti) }\end{array}$ & 0,00198916 & 0,01411344 & $\begin{array}{l}0,0061465 \\
6\end{array}$ & 0,00376996 \\
\hline 4 & $\begin{array}{l}\text { Thick-lipped catfish } \\
\text { (Cinetodus crassilabris) }\end{array}$ & 0,00338724 & 0,01411344 & $\begin{array}{l}0,0081360 \\
4\end{array}$ & 0,00516961 \\
\hline 5 & $\begin{array}{l}\text { Nurseryfish } \quad \text { (Kurtus } \\
\text { gulliveri) }\end{array}$ & 0,130321 & 0,13417569 & $\begin{array}{l}0,1568952 \\
1\end{array}$ & 0,13697401 \\
\hline 6 & $\begin{array}{l}\text { New Guinear Tiger } \\
\text { Perch } \\
\text { campbelli) }\end{array}$ & 0,00000225 & 0 & 0 & 0,000001 \\
\hline 7 & $\begin{array}{l}\text { Scale Croaker (Nibea } \\
\text { squamosal) }\end{array}$ & 0,00005329 & 0 & 0 & 0,00002304 \\
\hline 8 & $\begin{array}{l}\text { Barramundi (Lates } \\
\text { calcarifer) }\end{array}$ & 0,00017161 & 0 & 0 & 0,00007396 \\
\hline 9 & $\begin{array}{l}\text { Amoy } \\
\text { (Argyrosomus } \\
\text { amoyensis) }\end{array}$ & 0,00154449 & 0,00039204 & $\begin{array}{l}0,0009859 \\
6\end{array}$ & 0,00126025 \\
\hline 10 & $\begin{array}{l}\text { Striped threadfin } \\
\text { (Polydactylus plebeius) }\end{array}$ & 0,02852721 & 0,02509056 & 0,022201 & 0,026569 \\
\hline 11 & $\begin{array}{l}\text { Fourfinger threadfin } \\
\text { (Eleutheronema } \\
\text { tetradactylum) }\end{array}$ & 0,04515625 & 0,039204 & $\begin{array}{l}0,0325441 \\
6\end{array}$ & 0,04133089 \\
\hline 12 & $\begin{array}{lr}\text { Oxeye } & \text { Herring } \\
\text { (Megalops cyprinoides) }\end{array}$ & 0,00041616 & 0 & $\begin{array}{l}0,0000608 \\
4\end{array}$ & 0,00023409 \\
\hline 13 & $\begin{array}{l}\text { Chinese Herring } \\
\text { (Lebtobrama } s p)\end{array}$ & 0,00030625 & 0 & 0 & 0,00013225 \\
\hline 14 & $\begin{array}{l}\text { Flapnose ray } \\
\text { (Rhinoptera javanica) }\end{array}$ & 0,00003364 & 0 & 0 & 0,00001444 \\
\hline 15 & $\begin{array}{l}\text { Puffer fish (Marilyna } \\
\text { meraukensis) }\end{array}$ & 0,00001936 & 0,00039204 & $\begin{array}{l}0,0000608 \\
4\end{array}$ & 0,00004489 \\
\hline 16 & $\begin{array}{l}\text { Narrow-barred Spanish } \\
\text { mackerel } \\
\text { (Scomberomorus } \\
\text { commerson) }\end{array}$ & 0,00010404 & 0 & 0 & 0,00004489 \\
\hline 17 & $\begin{array}{l}\text { Freshwater Anchovy } \\
\text { (Thryssa scratchleyi) }\end{array}$ & 0,00030625 & 0 & $\begin{array}{l}0,0001392 \\
4\end{array}$ & 0,00020736 \\
\hline \multicolumn{2}{|c|}{ Total (C) } & 0,21249212 & 0,22748121 & $\begin{array}{l}0,2293882 \\
6\end{array}$ & 0,21622669 \\
\hline
\end{tabular}

The value of dominance index (Table 6) of the type of fish caught for three months in the Maro River, Gudang Arang, is 0,21622669. This result shows that, among the species found, there is no dominance. Furthermore, based on the overall species of fish caught, the highest dominance index value, in general close to 0, is found in Kurtus gulliveri in Station 3 with a dominance value of 0,15689521 .

\subsection{Physical Parameters and Water Chemistry}

The results of physical parameters, water chemistry, can be seen in Table 7 below:

Table 7. The Results of Physical Parameters and Water Chemistry

\begin{tabular}{|c|c|c|c|c|c|c|}
\hline \multirow[b]{2}{*}{$\begin{array}{l}\mathbf{N} \\
\mathbf{0}\end{array}$} & \multirow[b]{2}{*}{$\begin{array}{l}\text { Paramete } \\
\mathbf{r}\end{array}$} & \multirow[b]{2}{*}{ Unit } & \multicolumn{3}{|c|}{ Station } & \multirow{2}{*}{$\begin{array}{l}\text { Range } \\
\text { (ST1+ } \\
\text { ST2+ST } \\
\text { 3) }\end{array}$} \\
\hline & & & $\begin{array}{l}\text { I } \\
\text { (Estu } \\
\text { ary) }\end{array}$ & $\begin{array}{l}\text { II } \\
\text { (Floodg } \\
\text { ate) }\end{array}$ & $\begin{array}{l}\text { III } \\
\text { (Ca } \\
\text { pe) }\end{array}$ & \\
\hline 1 & $\begin{array}{l}\text { Temperatur } \\
\mathrm{e}\end{array}$ & ${ }^{0} \mathrm{C}$ & $\begin{array}{l}28 \\
29\end{array}$ & $29-31$ & $\begin{array}{l}29- \\
30\end{array}$ & $28-31$ \\
\hline 2 & $\begin{array}{l}\text { Flow } \\
\text { Velocity }\end{array}$ & $\mathrm{m} / \mathrm{dt}$ & 0,26 & 0,28 & 0,30 & $\begin{array}{l}0,26 \quad- \\
0,30\end{array}$ \\
\hline 3 & Salinity & Ppt & $\begin{array}{l}28,0 \\
30,0\end{array}$ & $\begin{array}{l}18,0 \\
20,0\end{array}$ & $\begin{array}{l}18, \\
0^{-}- \\
19,0\end{array}$ & $\begin{array}{l}18,0 \\
30,0\end{array}$ \\
\hline 4 & Water pH & & $7-8$ & $6-6,5$ & $\begin{array}{l}7- \\
7,5\end{array}$ & $6-8$ \\
\hline
\end{tabular}

\section{CONCLUSIONS}

There were 17 fish species caught in Maro River, Gudang Arang, Maro Village, Merauke Regency, namely: Kurtus gulliveri, Eleutheronema tetradactylum, Polydactylus plebeius, Cinetodus crassilabris, Cinetodus froggatti, Argyrosomus amoyensis, Mugil sp, Megalops cyprinoides, Thryssa scratchleyi, Lebtobrama sp, Lates calcarifer, Marilyna meraukensis, Scomberomorus commerson, Nibea squamosal, Rhinoptera javanica, Harpadon microchir, Datnioides Campbelli.

\section{REFERENCES}

1. Dinas Kelautan dan Perikanan Kabupaten Merauke, 2010. Jumlah Produksi Ikan. Dinas Kelautan Dan Perikanan Kabupaten Merauke.

2. Puslittan. 1986. Survey dan Pemetaan Tanah Tingkat Tinjau Daerah Merauke S. DigulPantai Kasuari, Propinsi Irian Jaya. Laporan Akhir No. 13/1986.

3. Dinas Pekerjaan Umum Kabupaten Merauke, 2008. Data Pengambilan Sampel Air Sungai Maro. Dinas Pekerjaan Umum Kabupaten Merauke.

4. Hendra Satria, 2007. Jenis ikan di sungai Maro. Jurnal Biologi Reproduksi ikan di Sungai Maro, Merauke, Propinsi Papua.

5. Kelurahan Maro Disrik Merauke Kabupaten Merauke, 2013. Data Jumlah Penduduk dan Batas Wilayah Kelurahan Maro Disrik Merauke Kabupaten Merauke.

6. Soehartono, Irawan. 1995. Metode Penelitian Sosial. Bandung : PT Remaja Rosda Karya.

7. Zikmund, William G., (1997). Business Research Methods. USA: Dryden Press. Hill, New York.

8. Mantra, Ida Bagoes dan Kasto. 1989. 'Penentuan Sampel' dalam Metode Penelitian Survai. Masri Singarimbun dan Sofian Effendi, eds. Edisi Revisi. Jakarta: LP3ES.

9. Allen, G.R., 1989. Freshwater fishes of Australia. T.F.H. Publications, Inc., Neptune City, New Jersey.

10. Allen, G.R. (1991). Fres hwater $f$ Fishes of New Guinea. Cristensen Research Institute, Madang Papua New Guinea. $268 \mathrm{p}$.

11. Dinas Kelautan dan Perikanan Kabupaten Merauke, 2009. Jumlah Produksi Ikan. Dinas Kelautan Dan Perikanan Kabupaten Merauke.

12. Royce, W. F. 1972. Introduction to the fisheries sciences. Academic Press, inc. New York. $351 \mathrm{p}$.

13. Krebs C. J. 1972. Ecology, the Experimental Analysis of Distribution and Abundance. Harper and Rows Publisher. 694 p.

14. Misra, R. 1968. Ecology Workbook. Oxford \& IBH Publishing Co. New Delhi BombayCalcutta. 244 p. 
15. Odum, E. P. 1996. Dasar - Dasar Ekologi. Gajah Mada University Press: Yogyakarta.

16. Setyobudiandi, I. dkk. 2009. Sampling dan Analisis Data Perikanan dan Kelautan. Terapan Metode Pengambilan Contoh di
Wilayah Pesisir dan Laut. Penerbit : FPIK Institut Pertanian Bogor.

17. Legendre, L dan P. Legendre, 1983. Numerical Ecology. Elsevier Scientific Publish Company. Amsterdam. Netherland. $419 \mathrm{p}$. 